\title{
Harry Graf Kessler und die >Ballets Russes، Plastische Körperkunst Nijinskys im Kontext der europäischen Moderne
}

Harry Graf Kessler war durch seine Herkunft und ebenso durch seinen Lebensstil Europäer. ${ }^{1}$ In den Jahren vor dem Ersten Weltkrieg intensivierten und verdichteten sich seine Reisen zwischen Paris, Weimar, Berlin, London, München und Wien. Es ist die Zeit, in der er das Netzwerk seiner Kunst-Vermittlungen nicht nur in der bildenden Kunst, sondern auch verstärkt in den aktuellsten Entwicklungen in Theater, Oper und Tanz entfaltet. Im Zentrum von Kesslers mäzenatischem und ästhetischem Interesse standen in den Jahren 1909-1914 die von Serge Diaghilew geleiteten `Ballets Russes $\diamond^{2}$ Die Aufführungen dieser BallettTruppe aus Russland waren ein europäisches Kunstereignis, das durch Diaghilews Geschick als Impresario seit der ersten Saison in Paris, 1909, und erneut mit jedem Programm in Gastspielen in ganz Europa gefeiert wurde. Kessler hatte an diesem Erfolg einen bedeutenden Anteil. ${ }^{3}$ Zahlreiche Einträge in seine Tagebücher bezeugen seine Begeisterung und sein Engagement. Seit seinem ersten Besuch der Aufführungen der 'Ballets Russes` war er von der ästhetischen Innovation überzeugt, die >die Russen (wie er Diaghilews Truppe in Briefen und im Tagebuch häufig bezeichnete) für Theater und Ballett der Moderne bedeuteten: eine neue Form des Gesamtkunstwerks, ein Zusammenwirken von Körper, Bewegung, Rhythmus, Bild und Musik - aus dem Geiste des Tanzes. Bereits nach seinen ersten Besuchen der 'Ballets Russes«-Saison in Paris 1909, nach einer Aufführung von "Cléopâtre und "Les Sylphides" notiert er in sein Tagebuch am 4. Juni 1909: "Alles in Allem [ist] dieses russische Ballett eine der merkwürdigsten und wertvollsten Erscheinungen unse-

1 Zur Biographie vgl. Peter Grupp, Harry Graf Kessler. 1868-1937. Eine Biographie. München 1995; Laird M. Easton, Der Rote Graf. Harry Graf Kessler und seine Zeit. Stuttgart 2005 sowie Harry Graf Kessler, Tagebuch eines Weltmanns. Hg. von Gerhard Schuster und Margot Pehle. Marbach a.N. 1988 [Ausstellungskatalog].

2 Richard Buckle, Diaghilev. London 1979; Sjeng Scheijen, Diaghilev. New York 2009.

3 Vgl. Harry Graf Kessler, Das Tagebuch 1880-1937. Hg. von Roland S. Kamzelak und Ulrich Ott. 9 Bde. und 2 CD-ROMs. Stuttgart 2004ff. 
rer Zeit; Leidenschaft und Raffinement, wie sie sonst nicht zusammen vorkommen. ${ }^{4}$ Und von Beginn seiner Betrachtungen über die Aufführungen der ’Ballets Russes` ist es der Tänzer Waslaw Nijinsky, der am intensivsten seine Aufmerksamkeit fesselt: »Nijinsky männlich, aber schön wie ein griechischer Gott. « ${ }^{5}$ Sofort sieht er in ihm das ideale Modell für einen Bildhauer: Es ist Kessler, der Auguste Rodin und Aristide Maillol auf Nijinskys Körperkunst hinlenkt.

Kessler begleitete die Produktionen der `Ballets Russes` von Beginn ihrer sensationellen Auftritte in Paris bis zu den Auflösungs- und Erneuerungsprozessen der Truppe in den 20er Jahren, bis zu Diaghilews "Tod in Venedig « 1929. ${ }^{6}$ Unterbrochen wird diese kontinuierliche Begegnung und teilweise intensive Kooperation nur durch die Jahre des Kriegs 1914-1918. Im Folgenden möchte ich mich auf die Begegnungen Kesslers mit den `Ballets Russes` in den Jahren vor dem Ersten Weltkrieg konzentrieren. In dieser Zeit stehen seine Beobachtungen der 'Ballets Russes< und seine Pläne im Kontext von zwei Projekten, die Kessler mit hohem Einsatz zu verwirklichen suchte: das Nietzsche-Denkmal in Weimar ${ }^{7}$ und die Idee und Gestaltung eines (dramatischen) Balletts für die >Ballets Russes - genauer: für Nijinsky - »Die Josephslegende«. Beide Projekte wurden durch je höchst komplizierte Fragen, Probleme, personelle und institutionelle Verwicklungen in der Realisierung beeinträchtigt. Prozesse, die Kessler im Tagebuch und in Briefen minutiös spiegelt. Und bei beiden Projekten handelt es sich um groß angelegte Gesamtkunstwerke, in deren Zentrum ein Gedanke stand, der für Kesslers Ästhetik, für seine Kulturidee einer Verbindung von Kunst und Leben zentral war: die Feier einer Kunst der Sinnlichkeit in einem Szenario, in dem Körper und Geist in Bewegung, Rhythmus und Form ästhetisch gestaltet sind. Philosophie und Bewegung oder mehr noch: eine Philosophie der Bewegung

4 Harry Graf Kessler, Das Tagebuch. Bd. 4 [1906-1914]. Hg. von Jörg Schuster. Stuttgart 2005, S. 574.

5 Ebd.

6 Vgl. Buckle, Diaghilev (wie Anm. 2), S. 538-541. Kessler schrieb in seinem Tagebuch über den Tod Diaghilews im Jahr 1929, dass es sei, als ob ein Teil seiner Welt eingestürzt sei (vgl. Harry Graf Kessler, Das Tagebuch. Bd. 9 [1926-1937]. Hg. von Sabine Gruber und Ulrich Ott. Stuttgart 2010). Es ist zugleich das Jahr von Hugo von Hofmannsthals Tod - nach dem tragischen Selbstmord seines Sohnes.

7 Vgl. dazu die genaue Beschreibung der Idee und der monumentalen Anlage, die Kessler mit Van de Velde plante, im Brief an Hugo von Hofmannsthal vom 16.4.1911. In: BW Kessler, S. $323 f f$. 
(als ästhetisches Ereignis) ist die Basis beider Gesamtkunstwerk-Projekte: Sie gelten dem Philosophen Nietzsche, dessen Denken »am Leitfaden des Leibes" für die Kunst und Kultur der Moderne wegweisend wurde, und dem Tänzer, in der biblischen Figur des Joseph, des »Tänzers und Träumers" (wie Thomas Mann ihn charakterisiert hat). Mit dem Vorhaben des Nietzsche-Denkmals in Weimar entwickelte Kessler die Idee einer Verbindung von Tempel und Stadion - eines monumentalen Fest-Spielraums, der nicht nur räumlich, sondern auch ideell zwischen Bayreuth und Hellerau angesiedelt war. Und mit der "Josephslegende" suchte Kessler seinen Traum eines Balletts zu realisieren, in dem raffinierte Sinnlichkeit und Spiritualität des Körperlichen sich verbinden. Es wäre reizvoll, beide Projekte vergleichend zu betrachten - zumal beide in einem interessanten, für die Zeit typischen Kontext der Körperkultur stehen: mit der Verbindung von Sport und Tanz zum einen ${ }^{8}$ und zum anderen mit dem umfassenden Konzept des Rhythmus, dessen Wirken in allen Künsten, in Tanz, Drama, Musik und ebenso in den plastischen Künsten, Skulptur und Architektur, die Ästhetik dieser beiden Gesamtkunstwerke prägen sollte. ${ }^{9}$

Es ist hier nicht der Raum, um dieses Thema, das einen detaillierten Durchgang durch die Prozesse und Verwerfungen der beiden künstlerischen Vorhaben erforderte, zu verfolgen. Zudem gibt es zu beiden Projekten bereits ausgezeichnete Forschung. ${ }^{10}$ Ich möchte mich hier deshalb auf Kesslers Ästhetik einer neuen Körperkunst - aus dem Geiste der 'Ballets Russes - konzentrieren. Im Mittelpunkt einer solchen zukunftsweisenden Tanzkunst steht dabei Waslaw Nijinsky: als Tänzer und als Choreograph.

8 Nicht nur im Stadion des Nietzsche-Denkmals waren Sportereignisse (nach antikem Modell) geplant; auch in das Ballett "Die Josephslegende" integrierte Kessler Sportszenen: Geplant war ein 'Boxer-Ballett, das - wie Kessler im Brief an Hofmannsthal vom 25. August 1912 schreibt - angeregt war durch die `Bogenschützen«-Szenen der `Ballets Russes` in »Fürst Igor" (vgl. BW Kessler, S. 356f.).

9 Vgl. Harry Graf Kesslers Essay »Kunst und Religion«, in dem er diese Grundgedanken entwickelt: Kunst und Religion. Die Kunst und die religiöse Menge. In: Pan 5, 1899, H. 3, S. $163-176$.

10 Vgl. Alexander Kostka, `Das Gesamtkunstwerk aller Sinner. Zu einigen Facetten der Beziehung zwischen Hugo von Hofmannsthal und Harry Graf Kessler. In: Harry Graf Kessler. Ein Wegbereiter der Moderne. Hg. von Gerhard Neumann und Günter Schnitzler. Freiburg i.Br. 1997, S. 135-153; und Ders., Harry Graf Kesslers Überlegungen zum modernen Kunstwerk im Spiegel des Dialogs mit Henry van de Velde. In: Harry Graf Kessler. Ein Wegbereiter der Moderne, S. 161-187. Zur "Josephslegende" vgl. Edition und ausgezeichnete Dokumentation in: SW XXVII Ballette - Pantomimen - Filmszenarien, S. 63-86, sowie (Entstehung und Zeugnisse), S. 392-500. 
Kesslers Eindrücke von den `Ballets Russes` werden rasch zu Fantasien über ein Projekt, ein Projekt der Moderne - in Kooperation mit Diaghilew und seinen Künstlern. Er sah in den Jahren 1909-1913/14, oft in mehrfachen Aufführungen und an unterschiedlichen Theatern Europas, alle Ballette; zuerst "Pavillon d'Armide«, über das er an Hugo von Hofmannsthal schrieb, es sei »[g]anz phänomenal «. ${ }^{11}$ Unermüdlich propagierte er dieses »Wunderbarste«, was er »in dieser Kunst «, ${ }^{12}$ außer der Ruth (gemeint ist Ruth St. Denis), ${ }^{13}$ gesehen habe, unter den Künstlern und Kunstkennern seines Umkreises. Hofmannsthal sucht er schon nach seinen ersten Erlebnissen des Tanz-Genies Nijinsky dafür zu gewinnen, ein Ballett für ihn zu schreiben: »Wenn du je ein Ballett schreibst (mit Strauss), müssen wir diesen jungen Nijinski bekommen. ${ }^{14}$ Und als Hofmannsthal es 1911 immer noch nicht geschafft hat, eine Aufführung zu sehen:

Nijinsky ist ein Genie, ein Genie ganz einziger Art, wie es unsere Zeit nicht gesehen hat, und wie wir es uns kaum in irgendeiner andren Zeit, außer vielleicht in der Antike, vorstellen können. Diese Verbindung von absoluter körperlicher Schönheit, Jugend und Grazie mit der äußersten gymnastischen athletischen Kraft und einer mimischen Gabe, die einem fortwährenden Dichten, einer fortwährenden genialen Schöpfung gleichkommt, muß man sehen; vorstellen kann man sich das, ohne es gesehen zu haben, absolut nicht. Ich sage da nicht nur, was ich empfinde, sondern was so verschiedene Naturen wie Maillol und Rilke spontan empfunden haben. ${ }^{15}$

Kessler brachte Nijinsky zu Maillol, als Modell der Apollo-Statue für das Nietzsche-Denkmal; er suchte Rilke für ein Ballett für Nijinsky zu interessieren - im Kontext der Begegnungen mit Rodin. Und Kessler notiert in seinem Tagebuch Rilkes Plan, unter dem Eindruck von Nijinskys "Licorne«, "ein Gedicht, jedenfalls Etwas Dramatisches ${ }^{16}$ zu schreiben. In diesem Geflecht von europäischen Künstler-Beziehungen, die Kessler um Nijinsky - als Stern einer neuen Tanzkunst - anordnete, kristallisie-

11 BW Kessler, Brief vom 28.5.1909, S. 233.

12 Ebd., S. 234.

13 Vgl. Gabriele Brandstetter, Der Traum vom anderen Tanz. Hofmannsthals Ästhetik des Schöpferischen im Dialog 'Furcht‘. In: Freiburger Universitätsblätter 30, 1991, H. 112: Hugo von Hofmannsthal: Dichtung als Vermittlung der Künste, S. 37-58.

14 BW Kessler, S. 234

15 Ebd., Brief vom 25.6.1911, S. 331.

16 Harry Graf Kessler, Das Tagebuch (wie Anm. 4), 14.7.1911, S. 708, vgl. auch Marbach Sonderausgabe, 1992, S. 10 (aus dem Nachlass Kesslers). 
ren sich zwei Muster seiner Kunstpolitik heraus, die wegweisend sind für die Moderne: erstens: Kessler als Diagnostiker des Publikums; und zweitens: Kesslers Ästhetik des Körpers im Kontext der Tanzmoderne.

Beide möchte ich, mit einem genaueren Blick auf die 'Ballets RussesWerke "L'Après-midi d'un Faune» (1911) und "Le Sacre du Printemps" (1913), ausführen.

\section{Kessler als Diagnostiker des Publikums}

In der Zeit der Saisons der `Ballets Russes` zwischen 1909 und 1914 ist Harry Graf Kessler nicht nur (wie auch insgesamt in der Kunst- und Theaterwelt) ein äußerst gebildeter Beobachter dieser Aufführungen, die er mit höchstem Kunstverstand, sicherem Gespür für das Neue und die spezifische Qualität der Darstellung und Form in seinen Tagebüchern und Briefen kommentiert. Zu diesem Prozess des ästhetischen Urteilens gehört auch seine Beobachtung der Beobachter. Seine präzise, oft pointierte Betrachtung der Betrachter wird nicht nur zu einem lebendigen Zeitspiegel, in dem die Begeisterungen und Abneigungen, die Urteile und Fehlurteile der Zeitgenossen, des bürgerlichen, des adligen, des intellektuellen und des snobistischen Kunstpublikums porträtiert sind. Kessler entwickelt mit und in diesen Skizzen über Meinungen und Kunstkontroversen zugleich ein Szenario für eine Ästhetik der Moderne, das diese wie mit einer doppelten Belichtung abbildet. Ja, man könnte sagen: Kesslers Schreiben über eine "Kunst des Publikums" - in der Tagebuch-Praxis ebenso wie in der Theorie, in seinem kurzen Essay "Kunst und Publikum « ${ }^{17}$ - nimmt aktuelle Theorien von Zuschauer und Publikum ${ }^{18}$ vorweg, die heute in der Theaterwissenschaft diskutiert werden. Kesslers Grundthese besagt, dass die Formen des Publikums in der Antike (und in den religiösen Ritualen alter Kulturen) - »die religiös erregte Menge ${ }^{19}$ - in der Moderne durch eine »Vielheit des Publikums « ${ }^{20}$

17 Vgl. Harry Graf Kessler, Kunst und Publikum. In: Die Neue Rundschau, XVII. Jahrgang der freien Bühne. Bd. 1. Berlin 1906, S. 112-116.

18 Vgl. Jacques Rancière, Der emanzipierte Zuschauer. Wien 2009; Gabriele Brandstetter, Figuration der Unschärfe. Der (un)beteiligte Betrachter. In: Texte zur Kunst 15, Juni 2005, H. 58, S. 74-79; Erika Fischer-Lichte, Die Entdeckung des Zuschauers. Paradigmenwechsel auf dem Theater des 20. Jahrhunderts. Tübingen/Basel 1997.

19 Harry Graf Kessler, Kunst und Publikum (wie Anm. 17), S. 113.

20 Ebd., S. 112. 
ersetzt seien. Die Vielzahl und Differenzierung der Kunstereignisse gehe einher mit einer Diversifizierung der Betrachter. So müsse man, laut Kessler, Publikum im Plural betrachten, nicht »das Publikum, nicht Ein Publikum, sondern ebenso viele Publika - ich kann leider kein anderes gleich anschauliches Wort finden - wie es Werke, Spieler, Lehrer gibt. ¿11 $^{21}$ Das Plädoyer, das aus diesem Befund folgt, zielt auf die Bildung der ästhetischen Wahrnehmung, damit die Sinnlichkeit der Kunst (und hier argumentiert Kessler nicht nur kunsttheoretisch und philosophisch, sondern auch mit den Erkenntnissen der psycho-physischen Wissenschaft, z.B. von Wilhelm Wundt) bewusst rezipiert werden könne. Nicht nur eine "Schule des Sehens" (wie Oskar Kokoschka formulierte), sondern gar eine "Gymnastik des Empfindens" müsse die Sinne der Betrachter „tränieren «22 (wie Kessler sagt, ganz bewusst mit einem Begriff aus dem Sport und der Körperkultur).

Wie zeigt sich nun aber die Praxis einer solchen Kunst des Publikums? Und wie beobachtet wiederum Kessler diese Spaltungen in den Vielheiten der >Publikar?

Das Tagebuch wird dabei zum >Publikations«-Raum, zum PublikumsOrt für die Spiegelungen unterschiedlicher Betrachterstandpunkte der ästhetischen Wahrnehmung. Besonders prägnante Beispiele dafür sind die beiden skandak-Ballette der 'Ballets Russes<, "L'Après-midi d'un Faune" und "Le Sacre du Printemps" - beide von Nijinsky choreographiert. Über die Aufführung des "Faun« in Berlin (an der Kroll-Oper) schreibt Kessler, wie Nijinsky auf ein geteiltes Echo stößt: »Stürmischer Erfolg des Faun, der wiederholt werden musste. Nur Bie drehte sich herum und flüsterte: `Daran finde ich Nichts. I Ich fast noch mehr als in Paris ergriffen.« ${ }^{23}$ Mit wenigen Zeilen kontrastiert Kessler seine persönliche Berührtheit, die Begeisterung des Publikums (zu dem rganz Berlin', d.h. Künstler und Kulturschaffende, zählt) und die Gegenposition von Oskar Bie - dem Kritiker-Papst, einem der größten Ballettkenner und TanzSchriftsteller dieser Zeit, der jedoch ganz konservativ der salten< Schule des klassischen Balletts anhing. ${ }^{24}$ Gegen Ende des Ballettabends, nach

21 Ebd.

22 Ebd., S. 115.

23 Harry Graf Kessler, Das Tagebuch (wie Anm. 4), 10.12.1912, S. 861f.

24 Vgl. Oskar Bie, Das Ballett. Mit 3 mehrfarbigen Kunstbeilagen und 14 Vollbildern in Tonätzung. Berlin 1905.

306 Gabriele Brandstetter 
Nijinskys Auftritt in dem Ballett "Carnaval« zur Musik Robert Schumanns, beobachtet Kessler die Verwandlung des Publikums: wie es sich "erheben und in die Traumwelt des Karnevals hineinstürzen« wollte. Nijinskys Genie habe, so Kessler, aus den "steifen Berlinern" ein "Neapolitanisches, südliches Publikum « ${ }^{25}$ gemacht.

Die Kritiken und Urteile von Theaterleuten und anderen Künstlern passieren im Tagebuch oft in kurzen Streiflichtern - und entwerfen das Spektrum eines vielfältigen Betrachter-Szenarios im Gegenüber der Aufführungen: so, wenn Sarah Bernhardt mitten im Gespräch mit Max Reinhardt, mit Kessler und D'Annunzio diskutiert und mit »der heftigsten Abneigung" von »den Russen« spricht, »deren Tanz kein Tanz, deren Dekorationen und Kostüme namentlich in der Farbe sbarbares', 'horribles`, infects` seien. Sie habe sich an der Brüstung ihrer Loge festklammern müssen, um nicht fortzulaufen. ${ }^{26}$ Damit ist nicht nur ein Geschmacksurteil von Sarah Bernhardts Eindruck von den 'Ballets Russes festgehalten; Kesslers kleines Porträt gibt auch die gestische Dimension, die exaltierte Situation wieder, in der dieses Kunsturteil zugleich zu einer Szene der Diva wird. Oft sind es solche kleinen, szenischen Skizzen und Porträts, die unterschiedliche Betrachter-Perspektiven aus jenen Kreisen, in denen Kessler sich bewegte, zu einem Kaleidoskop der 'Publikar der >Ballets Russes` fügen. So etwa, wenn Kessler zur Premiere von Nijinskys »Faun" (dessen Produktion und Rezeption er begleitet) notiert, wie er mit Jean Cocteau über den Artikel spricht, den Roger Marx im Namen von Rodin lancieren soll: Marx habe Rodins Aussprüche über

25 Harry Graf Kessler, Das Tagebuch (wie Anm. 4), 10.12.1912, S. 862.

26 Ebd., 28.5.1912, S. 832. - Die Äußerung Sarah Bernhardts ist insbesondere aufschlussreich in ihrem Urteil über die Kostüme: Denn die Kostüme von Léon Bakst führten in Paris, insbesondere nach "Sheherazade« (1911), zu einer regelrechten Mode-Welle à la ‘Ballets Russes‘, in Kreationen von Paul Poiret u.a. (vgl. Gabriele Brandstetter, Virtuoses Gesamtkunstwerk? Steigerung, Exzess und Unterbietung in der Ästhetik der 'Ballets Russes`. In: Genie - Virtuose - Dilettant. Konfigurationen romantischer Schöpfungsästhetik. Hg. von Ders. und Gerhard Neumann. Würzburg 2011, S. 179-198); dies scheint für den Geschmack von Sarah Bernhardt, die selbst eine Modeikone ihrer Zeit war, ein Affront gewesen zu sein. Vgl. Jane Pritchard (Hg.), Diaghilev and the Golden Age of the Ballets Russes 1909-1929. London 2010. 




Abb. 1: Georg Kolbe, Tänzer (Nijinsky), 1913/19. Bildarchiv Georg Kolbe Museum, Foto: Markus Hilbig 


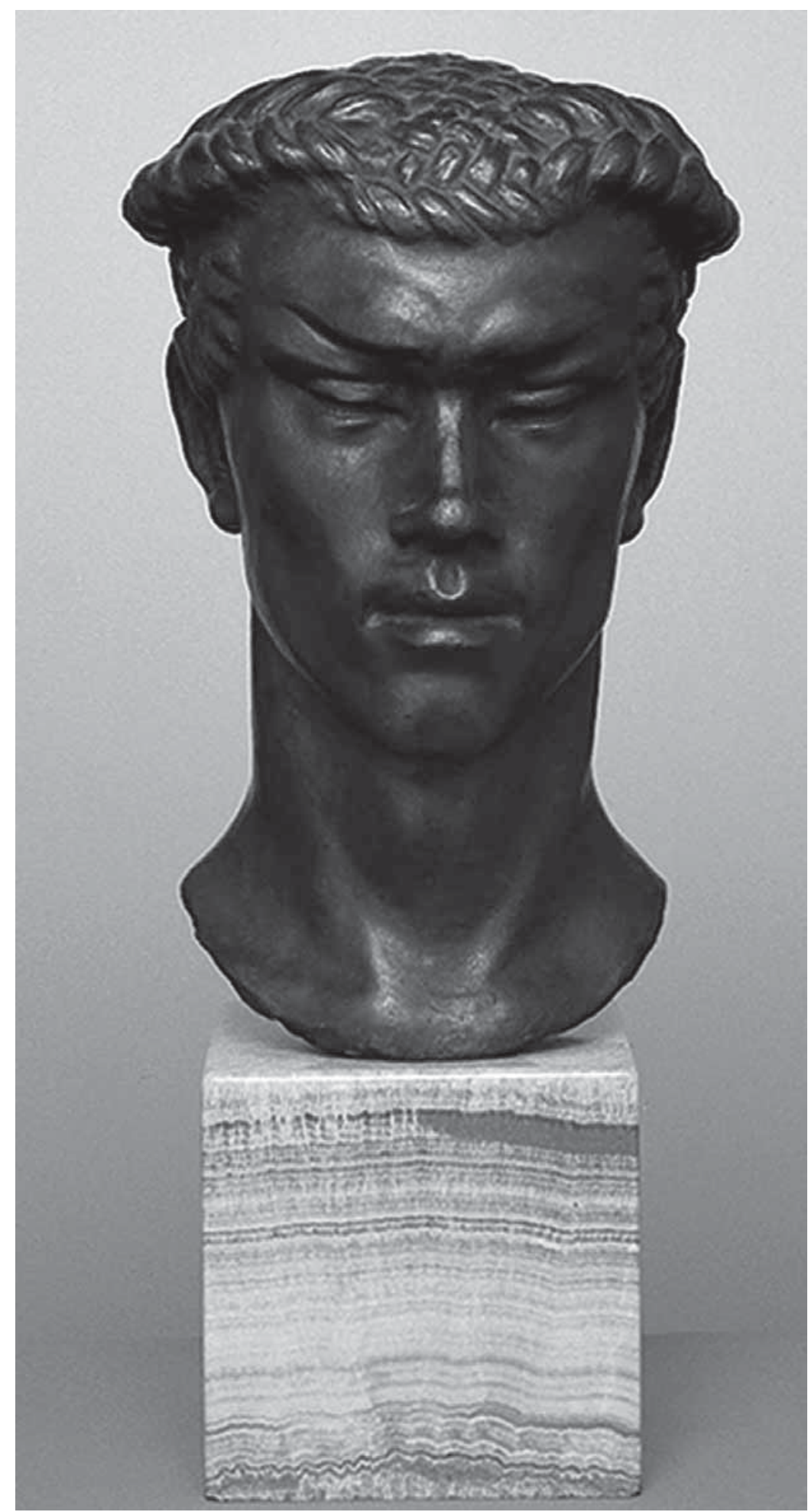

Abb. 2: Vincenza Una Troubridge, Vaslaw Nijinsky in "L'Après-midi d'un Faune«. Bronzebüste, signiert und datiert 1912/13, nummeriert 3/3, Höhe $32 \mathrm{~cm}$ 
"L'Après-midi d'un Faune« zu sammeln versucht, doch Rodin habe immer nur gesagt: „C'est de l'antique, c'est de l'antique «27 - und daraus lasse sich wohl schwer ein Artikel spinnen - worauf Cocteau meint, Marx werde das schon hinbekommen. Und danach wird Kessler zum aufmerksamen Chronisten der öffentlichen Kontroverse, die durch diesen Artikel im "Matin« und durch einen Gegenartikel des Chefredakteurs des "Figaro«, Gaston Calmette, der den »Faun« als "widerlich und obszön" einstuft, hervorgerufen wird. ${ }^{28}$

Während Kessler den Skandal um Nijinskys Darstellung des 'Faun mehr in seinen öffentlichen Formen der Zeitungsartikel und ihrer Protagonisten verfolgt - und zugleich in der `Hinterbühner dieser Szenen, nämlich im Atelier Rodins ${ }^{29}$-, berichtet er vom Aufruhr, den Nijinskys "Le Sacre du Printemps" verursachte, direkt aus dem Zuschauerraum. Zugleich gibt er aber auch Einblick in zwei weitere Betrachter-Szenarios, eines vor und eines nach der Premiere, so dass der vielfach dokumentierte Skandal von "Le Sacre du Printemps", den Gustave de Pavlovsky als "massacre du printemps « ${ }^{30}$ bezeichnete, relativiert wurde. Über den Aufruhr im Zuschauerraum, der von vielen Zeitzeugen, z.B. von Cocteau, Carl van Vechten, Valentine Gross-Hugo, farbig und höchst emotional beschrieben wurde, berichtet Kessler im Tagebuch nur knapp und trocken:

Das Publikum, das glänzendste Haus, das ich in Paris je gesehen habe, Aristokratie, Diplomaten, Halbwelt, war von Anfang an unruhig, lachte, zischelte, machte Witze; hier und dort standen Einige auf. Strawinski, der mit seiner Frau hinter uns sass, raste nach kaum fünf Minuten wie ein Besessener hinaus. Plötzlich rief aus der Galerie eine Stentorstimme: »Allons, les grues du Seizième (das $16^{\text {te }}$ Arrondissement, das der eleganten Welt), allez vous bientôt nous ficher la paix?" Die Antwort kam aus einer Loge »Les voilà ceux qui sont murs pour l'annexion.« Im selben Augenblick bekamen in Astrucs Loge d'Annunzio und Debussy Krakehl mit den Herren der Nebenloge, denen sie ins Gesicht schrieen: »Tas d’imbéciles.«Jetzt wurde der Lärm allgemein. Man

27 Harry Graf Kessler, Das Tagebuch (wie Anm. 4), 30.05.1911, S. 834.

28 Ebd., 30.05.1911, S. 835.

29 Vgl. ebd., 30.5./31.5.1911, S. 835-838.

30 Zit. nach François Lesure (Hg.), Igor Stravinsky. Le Sacre du printemps. Dossier de Presse. Press-Book. Genf 1980, S. 20; siehe auch Gabriele Brandstetter, Ritual als Szene und Diskurs. Kunst und Wissenschaft um 1900 - am Beispiel von Le Sacre du printemps. In: Konzepte der Moderne. Hg. von Gerhart von Graevenitz. Stuttgart/Weimar 1999, S. 367388, hier S. 367.

310 Gabriele Brandstetter 
hörte Astruc rufen: "Attendez la fin; vous sifflerez après" und als Antwort aus dem Parkett die Frage: "Dans combien de temps? « worauf Diaghilew replizierte: Dans cinq minutes. Pautrier hinter mir schrie: Qu'on leur joue le Tango; Marie Murat stritt sich laut mit ihrem Bruder. Gide, Ghéon die ganz Nouv Rev Franc stand wie die Phalanx im Logengang und bändigte durch Zurufe die Corbeille, die Logen der Polignacs, Rohans, Murats u.s.w. Und über diesen Höllenlärm giengen immerfort wie Sturmwetter Lachsalven und gegnerisches Klatschen, während die Musik wütete und auf der Bühne die Tänzer unentwegt und emsig prähistorisch tanzten. Am Schluss der Vorstellung schlug Welt und Halbwelt aufs Haupt, ehe frenetischer Beifall siegte, so dass sich Strawinski und Nijinski zeigen und immer wieder verbeugen konnten. ${ }^{31}$

In Kesslers Bericht wird der Raum des Publikums zu einer vielfältig gegliederten Topographie des Aufruhrs. Geradezu eine Choreographie und Szenerie von Aktion und Gegenaktionen verteilen sich hier im Verlauf der Vorstellung: Logen und Parkett, Galerien, Gänge und Balkon, segmentiert in soziale Schichten und Protagonisten des Fortschritts und der Tradition, der Mode und des Snobismus - zwischen Tango (der in Paris um 1913 der Modetanz war) und Ballett. Kessler greift einzelne Stimmen, Figuren, Aktionen und `Corps«-Bewegungen - wie z.B. die Phalanx der "Nouvelle Revue Française" im Logengang oder die Klatsch-Salven - auf und zeichnet damit die Dynamik im Auditorium als eine Art `Counter-Ballett zum 'Frühlingsopfer-Ritual auf der Bühne. ${ }^{32}$ Diese große Szene des Skandals wird nun gleichsam vor- und nachbereitet durch Kesslers Berichte von den Proben und Backstage-Ereignissen. Einen Tag vor (!) der Premiere berichtet er, dass er zur Probe von "Le Sacre du Printemps « ins "Théâtre des Champs Elysées" ging, die Probe zwar verpasste, danach aber "mit Diaghilew, Nijinski, Strawinsky, Ravel, Werth, Mme Edwards, Gide, Bakst, Oscar Fried usw. zu Larue « ${ }^{33}$ zum Dinieren ging, "wo allgemein die Ansicht herrschte, dass es morgen Abend bei der Premiere einen Skandal geben würde «. ${ }^{34}$

31 Harry Graf Kessler, Das Tagebuch (wie Anm. 4), 29.5.1913, S. 886.

32 Zur Interpretation der 'Rituak-Inszenierung in Strawinskys/Nijinskys "Le Sacre du printemps« vgl. Brandstetter, Ritual als Szene und Diskurs (wie Anm. 30).

33 Harry Graf Kessler, Das Tagebuch (wie Anm. 4), 28.5.1913, S. 885.

34 Ebd., S. $885 f$. 




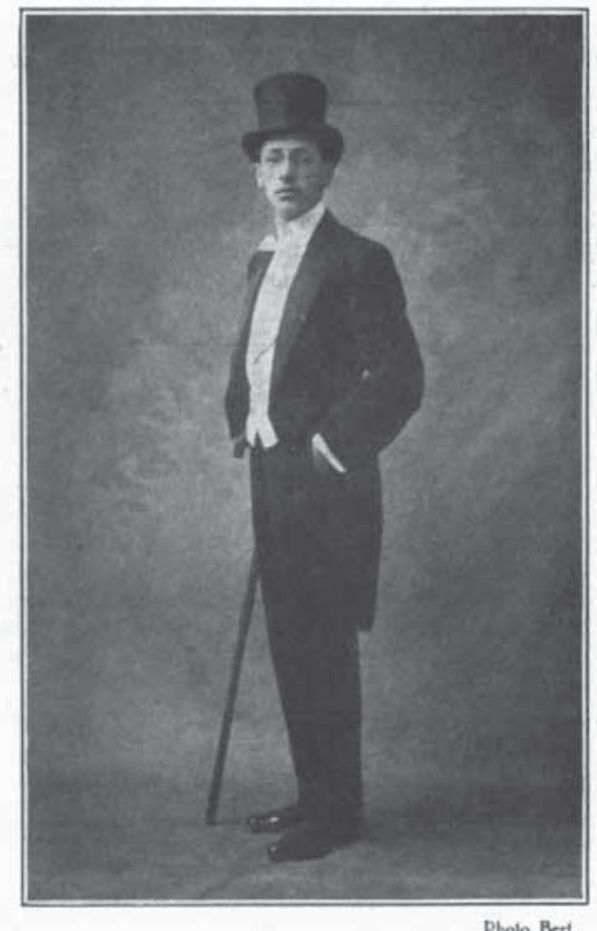

M. STRAWINSKY

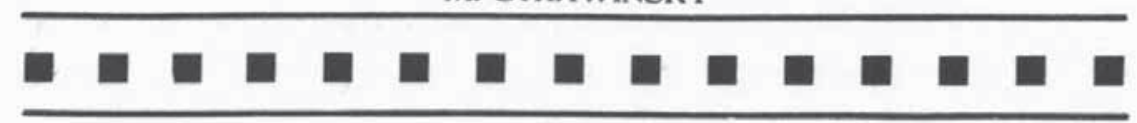

Abb. 3: Programm des Théâtre des Champs-Elysées, russische Saison, 29. Mai 1913 
Der Skandal der Uraufführung wird - durch diese Prämisse eines serwarteten Skandals - gleichsam zu einem Teil der Inszenierung und ihrer Propaganda. Umso mehr teilt sich die Szene und die Aufmerksamkeit eines Beobachters wie Kessler auf in die Vorstellung auf der Bühne und in das Beobachten der Beobachter - der Geschehnisse - im Publikum. Der Ausklang des Abends krönt gewissermaßen die unterschiedlichen sstagings dieser »Frühlingsweihe«: Zum `wilden` Tanz auf der Bühne und zu den wilden Ausschreitungen im Zuschauerraum kam nun, wie Kessler schreibt, nach dem Souper, um drei Uhr morgens

eine wilde Fahrt durch die nächtliche im Mondschein wie ausgestorbene Stadt, Bakst sein Taschentuch am Spazierstock wie eine Fahne schwenkend, Cocteau und ich hoch oben auf dem Dach des Autos, Nijinski in Frack und hohem Hut still vergnügt in sich hineinlächelnd. Der Morgen dämmerte, als mich die wilde, lustige Gesellschaft an meiner Tour d'Argent absetzte. ${ }^{35}$



Abb. 4: Sem (Georges Gousat), »Le Massacre du Printemps«, 1913

35 Ebd., S. 886f. 
Dieses Nach-Spiel hat Züge einer komödiantischen Coda zum tragischen Mittel-Teil mit dem Frühlingsopfer der Bühnen-Choreographie.

Das gesamte Ereignis freilich wird im Zeichen des >Wilden (jenes Adjektiv, das am häufigsten vorkommt in Kesslers Bericht) kommentiert: eine Idee des ssauvage<, die mit dem Opfer-Ritual des Balletts und den wilden Aktionen im Publikum zugleich auch das Wetterleuchten am Horizont des kommenden Kriegs erkennen lässt. In der Wortwahl von Kessler wird es zur »Beschreibung eines Kampfes « ${ }^{36}$ im Auditorium ebenso wie in den stetig zunehmenden Einträgen zu den politischen Spannungen und militärischen Aufrüstungen in Erwartung der Kriegserklärung.

\section{Kesslers Ästhetik des Körpers im Blick auf die 'Ballets Russes‘ und die Tanzmoderne}

Wie aber positioniert Kessler sein persönliches ästhetisches Urteil vor dem Hintergrund des Kunstgeschmacks und der Körperkultur-Ideen dieser Zeit? Kesslers Einschätzung von "Le Sacre du Printemps« zeigt seinen Blick für jene Qualitäten des Stücks, die es zu einem der radikalsten und wirkungsträchtigsten Ereignisse im Tanz des 20. Jahrhunderts machten. In nur wenigen Sätzen markiert Kesslers Eintrag die ästhetische Matrix von "Sacre«: "Eine ganz neue Choreographie und Musik; Nijinskis Tanzstil so verschieden von Fokines wie Gauguins Malerei von $[\ldots]$ (Delacroix). « ${ }^{37}$

36 Franz Kafkas »Beschreibung eines Kampfes" wurde etwa gleichzeitig im Band »Betrachtung" (1912) publiziert.

37 Hier hat, laut Kommentar der Tagebuch-Edition, Kessler den Namen 'Delacroix gestrichen (vgl. Harry Graf Kessler, Das Tagebuch [wie Anm. 4], S. 886). - Diesen Vergleich mit Gauguin verwendet Kessler auch im Brief an Hugo von Hofmannsthal vom 4. Juni 1913 (BW Kessler, S. 361): Hier streicht Kessler noch deutlicher heraus, dass diese Aufführung von "Sacre«" »denkwürdig" gewesen sei - in »Nijinskis grandioser und absolut neuer Kunst rhythmischer Massenbewegungen«. Und Kessler vergleicht das Verhältnis Nijinsky/Fokine mit 'Gauguin in Relation zu 'Bouguereau< - und weiter: "Ich habe die Empfindung, daß wir in Nijinskis choreographischer Phantasie Etwas ebenso überraschend Neues, Starkes, Revolutionäres vor uns haben wie etwa in der poetischen Phantasie eines Poe oder zeichnerischen eines Beardsley, gegenüber dem, was vor ihnen da war." (Ebd., S. 361) Zu Kessler und die französische Kunst vgl. Beatrice von Bismarck, Harry Graf Kessler und die französische Kunst um die Jahrhundertwende. In: Zeitschrift des deutschen Vereins für Kunstwissenschaft 42, 1988, H. 3: Sammler der frühen Moderne in Berlin, S. 47-62.

\section{Gabriele Brandstetter}


Eine durchaus neue Vision, etwas Niegesehenes, Packendes, Überzeugendes ist plötzlich da; eine neue Art von Wildheit in Unkunst und zugleich in Kunst: alle Form verwüstet, neue plötzlich aus dem Chaos auftauchend. ${ }^{38}$

Kessler markiert hier in knapper und präziser Weise, worin das Neue die 'Vision<, das 'Niegesehene - von "Sacre" bestand: in der Art und Weise, wie Musik und Choreographie formal und emotional spackend waren; in der Ambivalenz von 'Kunst rischer Darstellung und Performanz eines rituellen Vorgangs; sowie in einem ästhetischen Ikonoklasmus, in dem die Konzepte von Schönheit, Grazie und klassischer Form des Balletts (als Theaterkunst) zerstört, invertiert und durch andere Gestaltungen von Körper und choreographischem Raum überschrieben wurden.

In dem Vergleich mit Gauguin ruft Kessler ein ganzes Kulturmuster seiner Zeit hervor, das mit einem Diskurs um die Konstruktion des Primitiven aus dem Geist der Kunst und Ethnologie der Moderne einhergeht. ${ }^{39}$ Die Empfindung des Primitiven, des >Wilden als des fremden Eigenen zeigt sich in den Arbeiten Paul Gauguins in paradigmatischer Weise. Seine Malerei - und sein Leben in der fremden Kultur - verkörpert, was er selbst ein "Atelier der Tropen« nannte: ${ }^{40}$ Jene "Dunkelzone«, die in der Selbstreflexion der europäischen Moderne mit dem >Wilden<, >Primitiven`, dem "Prähistorischen" (so Kessler im Tagebuch am 29. Mai 1913) oder der "Prä-Existenz" (Hugo von Hofmannsthal) bezeichnet wurde: der >dark continent des Eigenen, im Abject des Anderen und seinen dunklen Riten. Kessler äußert sich in den wenigen Passagen, die er zu "Sacre" schreibt (da er in dieser Zeit zu sehr mit seinem eigenen Entwurf der "Josephslegende" beschäftigt ist) wenig zum splot<, zum Thema des Balletts. Die Fragen des Opfers, der Ritualität, werden kaum diskutiert, sondern eher verlagert: in die Analyse der Körperästhetik und der choreographischen Qualität von »Le Sacre du Printemps«. So erörtert er

38 Harry Graf Kessler, Das Tagebuch (wie Anm. 4), 28.5.1913, S. 886.

39 Genaueres dazu - im Kontext von »Le Sacre du Printemps« zu Kunst und Wissenschaft, zum Mythos des 'Fremden und zum Primitivismus, in: Brandstetter, Ritual als Szene und Diskurs (wie Anm. 30), S. 371ff. - Zum Thema des 'Primitiven und der Moderne vgl. u.a. Erhard Schüttpelz, Die Moderne im Spiegel des Primitiven. Weltliteratur und Ethnologie (1870-1960). Paderborn 2005; Nicola Gess, Primitives Denken. Wilde, Kinder und Wahnsinnige in der literarischen Moderne (Müller, Musil, Benn, Benjamin). München 2013; Burkhardt Wolf, Die Sorge des Souveräns. Eine Diskursgeschichte des Opfers. Zürich 2004.

40 Vgl. Brandstetter, Ritual als Szene und Diskurs (wie Anm. 30). 
in seinem "Vorwort an die Darsteller « der "Josephslegende ${ }^{41}$ die gleichberechtigte Verbindung von Musik und Tanz in Rhythmus und Ausdruck als Errungenschaft der Tanzmoderne seit Isadora Duncan und Ruth St. Denis. Und er bemerkt, in Hinsicht auf Nijinsky als Tänzer und Choreographen, dessen eigene Schöpfungen des »Faun« und vor allem

das grausige, urweltliche Opferfest in der 'Frühlingsweiher, stiessen mit unheimlicher Gestaltungskraft vor in Gebiete, die den Gegenpol bilden zu allem, was das klassische Ballett verherrlicht hat; sie rissen aus dem Urgrund der Seele, wo sich der Mensch vom Tier scheidet, dunkelste Begierden, Krämpfe, Schmerzen empor, die selbst der Musik verschlossen schienen. ${ }^{42}$

Kessler bewegt sich mit dieser Diagnose am scutting edge der Kulturtheorie und einer Ästhetik der Moderne im Jahr 1913 - jenem Jahr, in dem zu eben diesen Themen des 'Primitiven und der modernen Zivilisation soeben Sigmund Freuds »Totem und Tabu« erschienen war. In der bildenden Kunst waren es parallel die Bekenntnisse zum Neoprimitivismus, zu den 'Wilden - etwa im 'Blauen Reiter, wo u.a. David Burljuk und Franz Marc über die »Neuen Wilden« in Expressionismus und russischem Kubofuturismus schrieben. ${ }^{43}$

Vor allem aber bewegt sich Kesslers - wenngleich knappe - Einschätzung von "Sacre« auf Augenhöhe mit der bedeutendsten kunstkritischen Abhandlung zu »Le Sacre du Printemps«, die dieses Werk in seinem paradigmatischen Charakter für die (Tanz-)Kunst der Moderne analysiert: Jacques Rivières im November 1913 in der "Nouvelle Revue Française" publizierter Artikel "Le Sacre du Printemps «. ${ }^{44}$ Rivière beginnt seinen Essay mit dem Satz: "La grande nouveauté du Sacre du Printemps, c'est le renoncement à la ssaucer. ${ }^{45}$ - Die große Neuheit von Le Sacre $d u$ Printemps ist der Verzicht auf die sSauce «. ${ }^{46}$ Das kulinarische Element der "Sauce« wird im Weiteren zu einem Unterscheidungskriterium zur (be-

41 Vgl. SW XXVII Ballette - Pantomimen -Filmszenarien, S. 477-480.

42 Ebd., S. 479.

43 Vgl. Wassiliy Kandinsky/Franz Marc, Der Blaue Reiter. Originalausgabe 1912. Kommentierte Neuausgabe von Klaus Lankheit. München 2004; vgl. Felix Philipp Ingold, Der große Bruch - Rußland im Epochenjahr 1913. München 2000, S. $92 \mathrm{ff}$.

44 Vgl. den Abdruck in Lesure (Hg.), Igor Stravinsky (wie Anm. 30), S. 38-48.

45 Ebd., S. 38.

46 Jacques Rivière, Le Sacre du Printemps. Dt. Übers. von Charlotte Bomy und Margrit Vogt. In: Tanz über Gräben. 100 Jahre sLe Sacre du Printemps`. Eine Veranstaltung der Kulturstiftung des Bundes und des Zentrums für Bewegungsforschung an der Freien Universität Berlin, Programmheft zur Konferenz im Radialsystem. Berlin 2013, S. 8. 
reits veralteten) Kunst der Stimmung, des Impressionismus, des Atmosphärischen (etwa bei Debussy oder bei den Malern des Impressionismus) oder im Tanz einer Lö̈e Fuller. Dem stellt Rivière - ganz ähnlich wie Kessler in seiner Bemerkung über Nijinskys Gegen-Choreographie zum klassischen Ballett ${ }^{47}$ - den Purismus, die Härte, das Schnörkellose von »Sacre" gegenüber:

Das Werk ist vollkommen rein. Sauer und hart, wenn Sie so wollen; doch sein Geschmack wird von keinem Saft überdeckt, seine Struktur von keiner Kochkunst verändert oder verunreinigt. Dies ist kein "Kunstwerk « mit all den üblichen kleinen Tricks. Nichts Verschwommenes, nichts durch Schatten Verwischtes; keinerlei Schleier und keine poetischen Abmilderungen; keine Spur von Atmosphäre. Das Werk ist unbehandelt und unverfälscht, die einzelnen Teile bleiben roh, sie werden uns ohne Zusatz von Verdauungshilfe überliefert. Hier ist alles direkt, unberührt, klar und grob. ${ }^{48}$

Wie Kessler, der im Vorwort zur "Josephslegende « über die "Frühlingsweihe" sagt, sie stoße mit geradezu "unheimlicher Gestaltungskraft vor in Gebiete", die »den Gegenpol bilden zu allem, was das klassische Ballett verherrlicht «, ${ }^{49}$ so betont auch Rivière diese Kontraposition von "Sacre« zum traditionellen Ballett. Die Choreographie stehe in

keinerlei Beziehung mehr [mit dem] klassischen Tanz. Alles wird neu begonnen, alles wird hart erarbeitet, alles wird neu erfunden. Die Neuheit ist so brutal und so roh, dass wir dem Publikum das Recht nicht verwehren dürfen, sich dagegen aufzulehnen - ein Recht, von dem es nur allzu gründlich Gebrauch gemacht hat. ${ }^{50}$

Neu ist in "Sacre" die choreographische Anordnung der Bewegung der Tänzer im Raum. Und neu, »brutal« - »almost bestial«, wie Bronislawa Nijinska, die Schwester Nijinskys über die Proben schreibt ${ }^{51}$ - ist die antiballetthafte Arbeit mit dem Körper.

47 Vgl. Harry Graf Kessler, Vorwort an die Darsteller. In: SW XXVII Ballette Pantomimen - Filmszenarien, S. 479.

48 Rivière, Le Sacre du Printemps (wie Anm. 46), S. 8.

49 Vgl. Harry Graf Kessler, Vorwort an die Darsteller (wie Anm. 47), S. 479.

50 Rivière, Le Sacre du Printemps (wie Anm. 46), S. 8.

51 Vgl. Bronislava Nijinska, Early Memoirs. London/Boston 1982, S. 459. 




Abb. 5: Fotografie von Charles Gerschel, "Sacre du Printemps«, 1913, aus "Le Théâtre» vom 1. Juli 1913

Die Formation des Raums war durch Nicholas Roerichs Bühnenbild das einen rituellen Raum für das sagenhafte sprähistorischer slawische Frühlingsopfer darstellte - und durch Nijinskys korrespondierende choreographische Körper-Anordnung strukturiert.

Valentine Gross-Hugos Bilder und Skizzen vermitteln einen Eindruck von dieser Räumlichkeit, die in ihren Mustern absticht von den symmetrischen und ornamentalen Figurationen des Balletts: "Die gesamte Choreographie des 'Sacre ist von einer Asymmetrie durchsetzt, die das Wesen des Werks ausmacht«, schreibt Jacques Rivière. ${ }^{52}$ Dabei ergibt die Struktur der Bewegung, in klar und einfach strukturierten Formationen von Kreisen, Linie, Schlangenlinien, Doppelkreisen in Gegenbewegung, und geschlossenen Blöcken der Körper eine neue Dynamik des Aufeinandertreffens dessen, was Kessler »die Menge « ${ }^{53}$ nennt.

Roerichs Bilder - die heute, wie auch sein Bühnenbild und die Kostüme, folkloristisch und naiv wirken - inspirierten, wie Bronislawa Nijinska berichtet, Nijinsky:

52 Rivière, Le Sacre du Printemps (wie Anm. 46), S. 8.

53 Harry Graf Kessler, Kunst und Publikum (wie Anm. 17), S. 113.

\section{Gabriele Brandstetter}


'Now that I am working on Sacre, Vaslav went on, 'Roerich's art inspires me as much as does Stravinsky's powerful music - his paintings, The Idols of Ancient Russia, The Daughters of the Earth, and particularly the painting called, I think, The Call of the Sun. [...] Roerich has talked to me at length about his paintings in this series that he describes as the awakening of the spring of primeval man. In Sacre I want to emulate this spirit of the prehistoric Slavs. ${ }^{54}$

Roerichs Malerei war beeinflusst von Paul Gauguin und Puvis de Chavanne, mit deren Arbeiten er während eines längeren Paris-Aufenthalts bekannt geworden war. Insofern weist der Vergleich mit der Kunst Gauguins, den Kessler in Bezug auf die Choreographie von Nijinsky anstellt, auch auf die Verknüpfung von Raumgestaltung, Körperdarstellung, Farbigkeit und Reduktion von "Sacre" als Formprinzipien.

Mehr noch als die choreographische Struktur ist es die Darstellung des Körpers, die in Nijinskys Arbeit rantiballetthaft ‘ ist - und für Publikum und Tanzwelt einen Affront darstellte: Nijinskys "Crime against Grace ${ }^{55}$

Die Tänzer bewegten sich nicht nach den Regeln der >danse d'écoler des klassischen Balletts. Stattdessen: eingedrehte Füße, abgeknickte Köpfe, gewinkelte Arme, die Bewegungen eckig und abrupt, chorisch stampfend, in Repetitionen, laufend, stürzend, auf den Boden geworfen. Gustave de Pavlovsky schrieb in einer Rezension in der Zeitschrift „Comoedia« (31. Mai 1913):

C'est cette même impression que nous ressentons devant ces gestes saccadés d'automates préhistoriques, devant ces attitudes spontanées et irraisonnées que nous offre »Le Sacre du Printemps«, et tout cela, malgré les dissonances, donne une impression d'automaticité animale, de réflexes convulsifs d'un style très précis, d'un genre très délimité. Et n'est-ce point tout justement cette impression d'animalité, de réflexes instinctifs que voulaient nous donner les auteurs? ? $^{56}$

54 Vgl. Nijinska, Early Memoirs (wie Anm. 51), S. 449; zu Nicholas Roerich vgl.Jacqueline Decter, Nicholas Roerich, Leben und Werk eines russischen Meisters [in Zusammenarbeit mit dem Nicholas Roerich Museum]. Basel 1989.

55 Vgl. Millicent Hodson, Nijinsky's Crime against Grace. Reconstruction Score of the Original Choreogaphy for Le Sacre du Printemps. Stuyvesant/New York 1996.

56 Gustave de Pavlovski, Le Sacre du Printemps. In: Lesure (Hg.), Igor Stravinsky (wie Anm. 30), S. 18-20, hier S. 20. 
Die brennende Frage nach einer randeren $<$ Ästhetik des Körpers im Tanz, nach einem Aufbruch in eine Ausdrucksdimension, die die Schönheitsmuster des traditionellen Balletts verlässt und die zugleich doch ganz aus der Bewegung und nicht aus dem Pantomimischen schöpft, treibt auch Kesslers Reflexion über die 'Ballets Russes‘. Nijinsky verkörpert diese neue Ästhetik. Die Verwandlungskraft seiner körperlichen Darstellung, die Kühnheit seiner Bewegungs(er)findung vergleicht Kessler mit der Kunst der Plastik - und zugleich, ähnlich wie Rivière und Gustave de Pavlovsky- mit einer genialen körperlichen Evokation und Reflexion der Grenze von Mensch und »animal«. So schreibt Kessler nach der Aufführung des "Faun« in Berlin im Tagebuch, er sei fast noch mehr ergriffen als in Paris:

Der Faun: das Tier, das den Menschen ahnt, das ahnt, was es heisst, Mensch zu sein, ein Tier das vergleicht, das sich und die andren vergleicht, erstes Aufflammen der Reflexion und damit der Tragik und auch der Komik; aber die Tragik überwiegend. Man meint, in allen Bewegungen von Nijinski zu fühlen, dass es ein Tier ist, das sich seiner Nacktheit schämt (Sündenfall). Äusserste Konzentration des Ausdrucks; eine solche Gewalt in der Sparsamkeit der Mittel erreicht nur das Genie. ${ }^{57}$

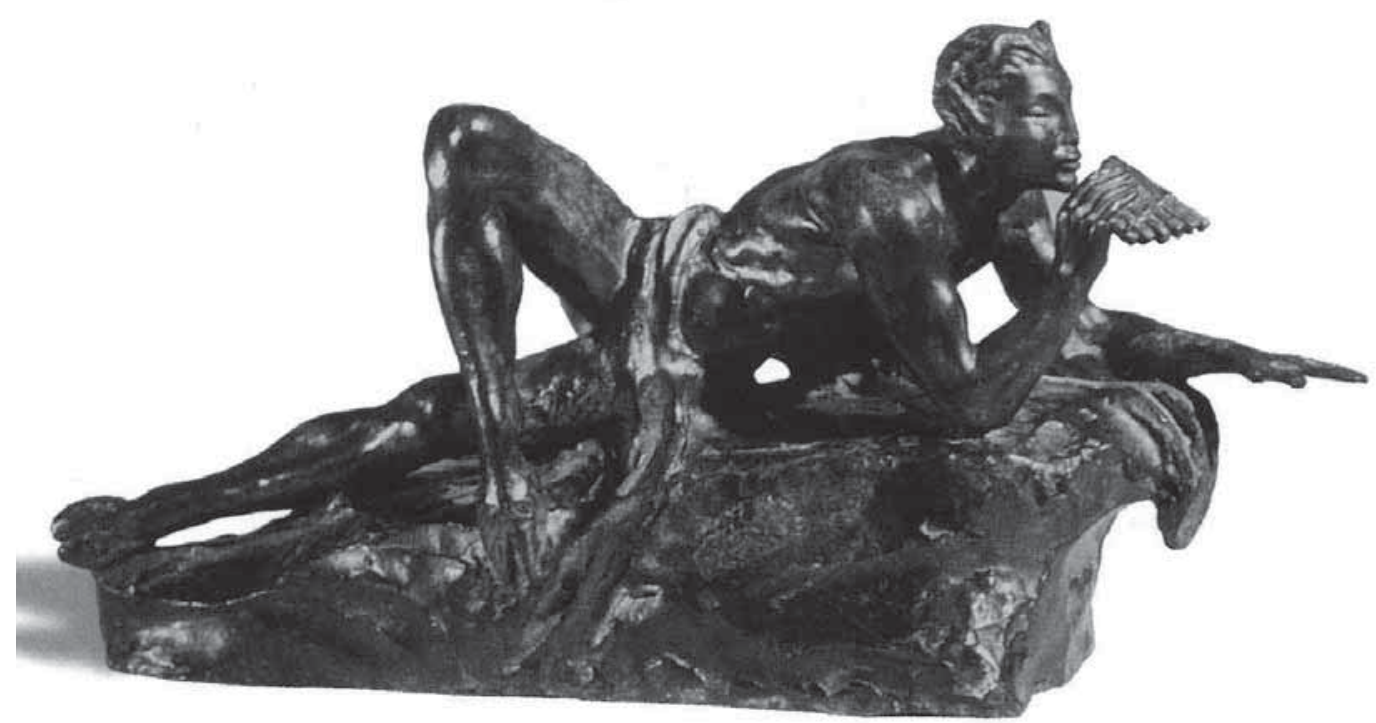

Abb. 6: Malvina Hoffman, Nijinsky als Faun. Bronze, ca. 1912

57 Harry Graf Kessler, Das Tagebuch (wie Anm. 4), 10.12.1912, S. 861f., hier S. 862

320 Gabriele Brandstetter 
Dieses Moment der Transgression, aus dem »Urgrund der Seele, wo sich der Mensch vom Tier scheidet « ${ }^{58}$ umkreist Kessler in seinen Fragen nach dem Neuen, nach dem Fremden in der Körperlichkeit Nijinskys immer wieder. So berichtet er auch, anlässlich der Vorstellungen der 'Ballets Russes in London, im Februar 1913, bei Gelegenheit eines Tees bei Lady Speyer, ${ }^{59}$ wie diese "mit einer merkwürdigen Mischung von Abscheu und Bewunderung von Nijinsky ${ }^{60}$ und seinem "Faun« sprach. "Er sei zweifellos ein Genie, aber gleichzeitig auch eine Art von Monstrum. Im Faun habe er in ihr Seiten berührt, vor denen sie sich fürchte: die tierischen, die ungeklärten, dunklen Triebe. ${ }^{61}$ Kessler diagnostiziert diese Doppelseitigkeit - zwischen "Monstrum" und "Genie«, zwischen Bewunderung und Abscheu - als eben jenes Kriterium des Modernen, das das Publikum spaltet. ${ }^{62}$ Eben diesen Kunststreit - in den Fragen einer neuen Ästhetik, die lebendig, sinnlich, körpernah ist - hält Kessler in seinen Tagebüchern fest: in seinen Beobachtungen der 'Ballets Russes erscheint diese Thematik wie ein roter Faden.

Worin besteht die Form des Körpers, im Tanz, in der Plastik, in Proportion, Volumen, Schwere, Verwandlungen und Modellierungen von Oberflächen und Bewegungen? Es ist eine Frage, die Rilke mit Blick auf Rodin erörtert. Und die Kessler in den Arbeiten mit und im Atelier Maillols beschäftigen. Eine Auseinandersetzung zwischen Misia Edwards, Kessler und Waslaw Nijinsky wirft Licht auf diese Kunst der

58 Ders., Vorwort an die Darsteller (wie Anm. 47), S. 479. - Zu Kesslers Konzept der "Josephslegende", in der Kooperation mit Diaghilew und Nijinsky einerseits und mit Hugo von Hofmannsthal und Richard Strauss und deren divergierenden ästhetischen Konzepten andererseits vgl. die umfassende Studie von Günter Schnitzler, Hofmannsthal, Kessler, Strauss: Die "Josephslegende«. Intermedialität und divergierende ästhetische Konzepte. In: Der Tanz in den Künsten 1770-1914. Hg. von Achim Aurnhammer und Günter Schnitzler. Freiburg i.Br. 2009, S. 307-344.

$59 \mathrm{Zu}$ Harry Graf Kesslers Mäzenatentum (und seinem Verkehr im Kreise von Mäzenen) vgl. Hildegard Nabbe, Mäzenatentum und elitäre Kunst. Harry Graf Kessler als Schlüsselfigur für eine kulturelle Erneuerung um die Jahrhundertwende. In: DVjs 64, 1990, H. 4, S. $652-$ 679 .

60 Harry Graf Kessler, Das Tagebuch (wie Anm. 4), 22.2.1913, S. 868.

61 Ebd.

62 So ergänzt er, auch im Blick auf die Konzeption der "Josephslegende« und aus der Argumentation seines Essays über "Kunst und Religion" heraus, im oben genannten Tagebucheintrag: "Zweifellos umgiebt Nijinsky namentlich seit dem 'Faun ausserhalb eines kleinen Kreises von Künstlern und Bewunderern überall diese Atmosphäre des Abscheus und der Furcht. In Wirklichkeit verträgt die Masse, vor Allem die kulturelle Masse, lebendige Kunst ebensowenig wie lebendige Religion; wo unwiderstehliches Genie sie zwingt, Kunst, die noch nicht sterilisiert und tot ist ins Auge zu fassen, hasst sie und entsetzt sich.« (Ebd.) 
Körperplastik: Am 20. Juni 1913, kurz nach der Premiere von »Le Sacre du Printemps«, diskutieren sie beim Souper über Maillol: »Die Edwards gegen seinen dicken Frauentypus Nijinski ergriff lebhaft für Maillol Partei. « ${ }^{33}$ Misia Edwards wendet ein, weshalb Maillol nicht schöne Frauen darstelle. "Pourquoi lui fautil des monstres? ${ }{ }^{64}$ Nijinsky stritt dafür, dass gerade diese breiten, schweren Formen neue Proportionen, neue Linien und Massen ergäben: "Cela Sacre du Printemps. Alors vous pas aimez Sacre du Printemps. $\ll^{65}$
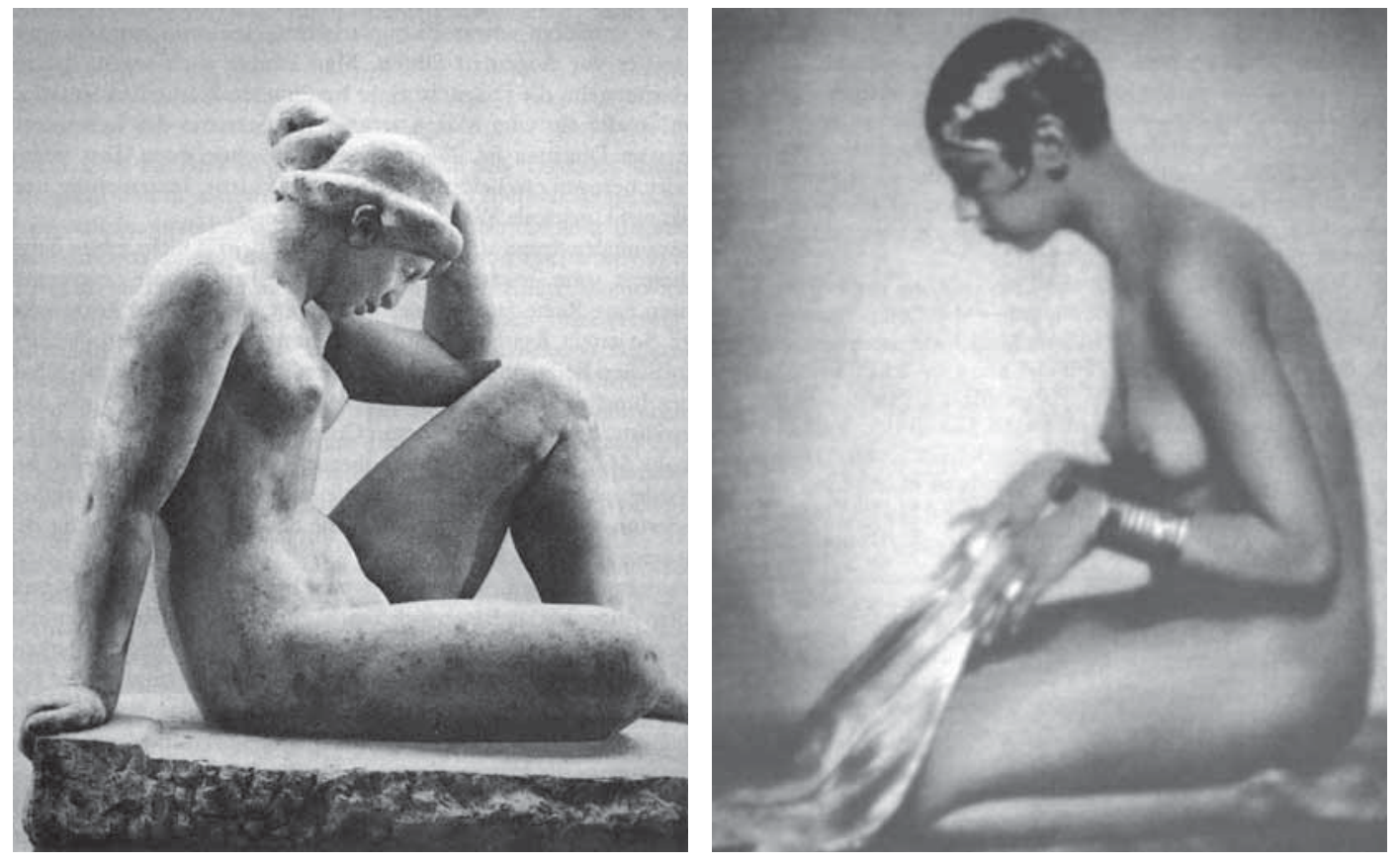

Abb. 7 und 8: Aristide Maillol, »La Méditerranée«, zwischen 1923 und 1927 (links); Josephine Baker, kauernd (rechts)

Es ist eben dieser Umgang mit dem Körper als plastischem, als räumlichem Material, das die Ästhetik dieses Meisterwerks der Moderne prägt. Kessler erkennt Nijinsky als den Skulpteur eines neuen Tanzes; und er porträtiert ihn nicht nur als den Tänzer, der - im »Faun« und in anderen Rollen - diese neue Gestalt von Bewegung verkörpert, sondern auch als den Choreographen und Bildhauer, der Körper in ihrer Materialität herausfordernd neu gestaltet. Eine analoge Gegenüberstellung von Maillols

63 Ebd., 20.6.1913, S. 897.

64 Ebd.

65 Ebd. 
Plastik und Tänzerkörper - als Dialog einer Bewegungs-Sinnlichkeit beschreibt Kessler etwa zehn Jahre später (1926) in der Begegnung mit Josephine Baker.

Am 24. Februar 1926 hat Kessler eine Gesellschaft von Künstlern und Intellektuellen zu sich nach Hause eingeladen - darunter auch Josephine Baker, deren Tanzkunst er bewundert und für die er ein Ballett (für die Bühne Max Reinhardts) plant, wiederum ist es, wie schon für Nijinsky als Joseph<, ein biblisch-exotisch-erotisches Thema, nämlich: Motive aus dem Hohenlied Salomos. ${ }^{66}$ Zunächst ist Baker zurückhaltend; dann taut sie auf - und tanzt eine Art Dialog mit Maillols großer sitzender FrauenPlastik "Méditerranée«, die in Kesslers Salon steht: "Dann machte sie einige Bewegungen, stark und ausdrucksvoll grotesk, vor der grossen Maillol-Figur. Offenbar setzte sie sich mit dieser auseinander. « ${ }^{67}$

Und Kessler beschreibt detailliert die Modellierungen, Posen, die Wucht der Plastik und die "grotesk grandiosen Bewegungen der Baker", die am Körper der Statue mehr interessiert war als an der illustren Künstler-Runde. "Genie sprach zu Genie«, ${ }^{68}$ ist Kesslers Fazit - ähnlich wie in der Szene von Nijinsky, Maillol und Misia Edwards. Es ist jene ganz seiner Idee folgende (Proben-)Arbeit, von der es Berichte gibt, wie Nijinsky hart und fordernd das Ungewohnte dieser Bewegungen mit den Tänzern der 'Ballets Russes< trainierte. ${ }^{69}$

Zwischen Tier und Puppe: Jacques Rivière beschreibt Nijinskys NeuKalibrierung des Körpers treffend:

Er [Nijinsky] nimmt seine Tänzer, arrangiert und dreht ihre Arme, er würde sie sogar zerbrechen, wenn er es wagte; er bearbeitet ihre Körper mit gnadenloser Brutalität, als seien sie Dinge; er verlangt ihnen unmögliche Bewegungen ab, Haltungen, die naturwidrig erscheinen. Doch dient dies dazu, ihre Ausdrucksmöglichkeiten bis ins Letzte auszuschöpfen. Und tatsächlich sprechen die Körper am Ende. Diese bizarren und gewaltsamen Formen machen etwas Unbekanntes sichtbar; sie stellen deutlich tausende komplizierte und geheimnisvolle Dinge dar, die wir nur noch anzuschauen haben. ${ }^{70}$

\footnotetext{
66 Vgl. Harry Graf Kessler, Das Tagebuch. Bd. 9 (wie Anm. 6), 13.2.1926, S. 727f.

67 Ebd., 24.2.1926, S. 738ff., hier S. 739.

68 Ebd.

69 Vgl. Nijinska, Early Memoirs (wie Anm. 51), S. 459.

70 Rivière, Le Sacre du Printemps (wie Anm. 46), S. 8.
} 
Eine Auseinandersetzung, die eben diese kontroverse Ästhetik im Umgang mit individuellem Körperausdruck einerseits und Arbeit mit dem Tänzer-Körper als `Material, als Marionette andererseits zum Inhalt hat, berichtet Kessler von einem Frühstück mit Nijinsky, Edward Gordon Craig und dem Komponisten Vaughan Williams (den Craig für die Komposition der "Josephslegende" vorgeschlagen hatte). ${ }^{71}$ Schon früher hatte Craig geäußert, dass er den "Faun" nicht möge, "weil die Figuren tot seien « ${ }^{72}$ In einer Diskussion über das Verhältnis von Regisseur und Schauspieler pointiert Craig zwei extreme Standpunkte: Individualität oder »totes Material«, wie "Marionetten«, und er betont, dass er (der Schöpfer der »Übermarionette«) heute das Erstere, also den individuellen Ausdruck, bevorzuge. "Nijinsky erwiderte er wolle lebendige Schauspieler, aber sie so lange bearbeiten bis sie wie totes Material gehorchten . $^{73}$ - Und Kessler endet die Szene mit der Bemerkung, dass eine Einigung nicht zu erzielen war: eine offene Kontroverse.

Nijinsky hat - eben mit diesem Prinzip, als Choreograph - in "Sacre» jene Neuartigkeit tänzerischer Darstellung inauguriert, die Jacques Rivière als eine Art neuer Grazie, eine Grazie nach dem Bruch mit den Ballettideen von Anmut und Schwerelosigkeit, akzentuiert: Eine Grazie, die man in der Profilansicht der Gesichter finde (jenem Körperkonzept, das Nijinsky für den »Faun" erfindet und auch in "Sacre" einsetzt), »in den geöffneten und steifen Händen, im Beben ${ }^{74}{ }^{74}$ Und er verstärkt dies noch im Tanz der auserwählten Jungfrau: der Tanz des Opfers - »in ihren kurzen, verfehlenden Zuckungen, in ihrer Verlegenheit, der schrecklichen Erwartung, in seiner gezwungenen und entstellten Gangart und in dem in den Himmel gereckten Arm als Zeichen von Anrufung, Bedrohung und Schutz. «75

Zum Schluss also: Das Opfer. Es ist auffallend, dass Harry Graf Kessler das Thema des Opfers, das »Le Sacre du Printemps« beherrscht, nicht direkt anspricht. In seinen Bemerkungen zur Fremdheit der Form, zum Transgressiven eines »urweltlichen" grausigen Opferfestes, zur Psychologie des Verdrängten, zwischen Tier und Mensch, den Kulten, und

71 Vgl. Harry Graf Kessler, Das Tagebuch (wie Anm. 4), 23.2.1913, S. 869.

72 Ebd.

73 Ebd.

74 Rivière, Le Sacre du Printemps (wie Anm. 46), S. 8.

75 Ebd.

\section{Gabriele Brandstetter}


»Krämpfen ${ }^{76}$ rührt er aber daran. - Und schließlich läuft in seinen Tagebucheintragungen, parallel zu den Kunstbegegnungen und den ästhetischen Debatten, eine kontinuierliche Beobachtung der politischen Spannungen in Europa - in die schon sehr früh eigene Kriegsvorbereitungen eingepasst sind: Während Kessler rastlos die Produktion, die komplizierte Kooperation zur "Josephslegende« zwischen Richard Strauss, Hofmannsthal, Diaghilew, Nijinsky (und später Léonide Massine) vorantreibt, beginnt er im Sommer 1913 bei seinem Regiment in Potsdam mit militärischen Übungen; und er notiert in kurzen Tagebucheinträgen: 25. Juni 1913 »im Auto nach Potsdam wo meine Übung angetreten. Bei der dritten Schwadron (Köckeritz).«77 Oder 2. August 1913: »Frühst. Heymel. Exerzieren auf Feld. "78 $^{78}$ 26. Juni 1914 notiert Kessler: "Schlechte Nachrichten. Österreich soll Serbien den Krieg erklärt haben. ${ }^{79}$ Es ist "[e]in Tanz über Gräben. ${ }^{80}$ Begonnen hat dieser Tanz freilich schon früher und die Atmosphäre der kommenden Katastrophe - und ihrer ungezählten Opfer - trug sich auch in die sensiblen Bildwelten der Kunst ein. Harry Graf Kessler hat auch diese Sorge - dass der Körper der Kunst und der von ihm am meisten geschätzten Künstler der `Ballets Russes das Opfer sein könnte - schon früh formuliert. In einem Brief an Hofmannsthal vom 10. November 1912 schreibt Kessler als Postskriptum:

Die Spannung zwischen Oesterreich und Serbien sieht von hier sehr beunruhigend aus. Sollte es diesmal wirklich zum Weltkrieg kommen? Und was wird dann aus Joseph? Wenn Ihr uns nur nicht den Nijinski abschießt $!^{81}$

76 Vgl. Harry Graf Kessler, Vorwort an die Darsteller (wie Anm. 47).

77 Ders., Das Tagebuch (wie Anm. 4), 25.6.1913, S. 900.

78 Ebd., S. 904.

79 Ebd., S. 916.

80 Vgl. Modris Eksteins, Tanz über Gräben. Die Geburt der Moderne und der Erste Weltkrieg. Reinbek bei Hamburg 1990.

81 BW Kessler, S. 360. 


\section{Abbildungsnachweise}

Abb. 1: Georg Kolbe, Tänzer (Nijinsky) 1913/19. Bildarchiv Georg Kolbe Museum, Foto: Markus Hilbig

Abb. 2: Vincenza Una Troubridge, Vaslaw Nijinsky in "L'Après-midi d'un Faune«. Bronzebüste, signiert und datiert 1912/13, nummeriert 3/3, Höhe 32 $\mathrm{cm}$. Theatre Museum London, Stiftung John Neumeier - Dance Collection Abb. 3: Heidy Zimmermann/Hermann Danuser (Hg.), Avatar of Modernity. The Rite of Spring Reconsidered. London: Boosey \& Hawkes, Program of the Theatre des Champs-Elysee, Saison russe, 29. May 1913, S. 417

Abb. 4: Nijinsky: legend and modernist - the dancer who changed the world. Katalog, Dans museet 2000, S. 105, Sem (Georges Goursat), »Le Massacre du Printemps«, 1913

Abb. 5: Nijinsky's Crime Against Grace. Reconstrucion of the Original Choreography for Le Sacre du Printemps. By Millicent Hodson. New York 1996, S. 106, Foto: Reconstruction of Nijinsky's Le Sacre du Printemps by Millicent Hodson and Kenneth Archer (1987)

Abb. 6: Nijinsky: legend and modernist - the dancer who changed the world. Katalog, Dans museet 2000, S. 99, Malvina Hoffman, Nijinsky as the Faun, bronce, ca. 1912

Abb. 7: Harry Graf Kessler: Ein Wegbereiter der Moderne. Hg. von Gerhard Neumann und Günter Schnitzler. Freiburg i.Br. 1997, darin: Gerhard Neumann, Wahrnehmungswandel um 1900. Harry Graf Kessler als Diarist, S. 54 Abb. 8: Harry Graf Kessler: Ein Wegbereiter der Moderne. Hg. von Gerhard Neumann und Günter Schnitzler. Freiburg i. Br. 1997, darin: Gerhard Neumann, Wahrnehmungswandel um 1900. Harry Graf Kessler als Diarist, S. 55 